http://sciforum.net/conference/ece-1

Conference Proceedings Paper - Energies „, Whither Energy Conversion? Present Trends, Current Problems and Realistic Future Solutions"

\title{
Evaluation of the Wave Energy Conversion Efficiency in Various Coastal Environments
}

\author{
Eugen Rusu ${ }^{1} *$
}

1 Department of Mechanical Engineering, "Dunarea de Jos" University of Galati, 111 Domneasca Street, 800201 Galati, Romania; E-Mails: erusu@ugal.ro (E.R.)

* Author to whom correspondence should be addressed; E-Mail: erusu @ugal.ro (E.R.); Tel.: +40-740-205-534; Fax: +40-236-461-353.

Received: 11 December 2013 / Accepted: 15 February 2014 / Published: 14 March 2014

\begin{abstract}
The main objective of the present work is to assess the performances of various WEC types that would operate in the nearshore. Three different groups of coastal environments were considered. They are: the western Iberian nearshore, two archipelagos (Canaries Islands and Madeira) and the sea environment. The most representative existent wave converters are evaluated in the analysis. In order to estimate the electric power expected in a certain location, the bivariate distributions of the occurrences corresponding to the sea states, defined by the significant wave height and the energy period, were designed in each coastal area. The wave data were provided by hindcast studies performed with numerical wave models or based on measurements. The transformation efficiency of the wave energy into electricity is evaluated via the load factor and also through an index defined as the ratio between the electric power estimated to be produced by each specific WEC and the expected wave power corresponding to the location considered. The work provides valuable information related to the effectiveness of various technologies for the wave energy extraction that would operate in different coastal environments. Moreover, the results can be extrapolated to other areas.
\end{abstract}

Keywords: wave power, WEC, electricity, efficiency, nearshore, wave models 


\section{Introduction}

In the medium to long term, wave energy has been identified with the potential to offer a significant contribution to the European and global energy system. At present, the technologies are still in early stages of development, but the evolution of the wave energy converters is very dynamic and major technology improvements are now expected in order to make the wave energy economically viable.

Since more than $70 \%$ of the Earth's surface is covered by ocean, the theoretical resource of ocean energy has been estimated at about four times the global electricity demand [1].

This trend of increasing the renewable energy extraction is also highly motivated by the global awareness related to the need of a transition to a lower-carbon energy system, since greenhouse gas emissions are recognized factors in the climate changes. The de-carbonization of the energy sector due to low carbon technological innovation is fundamental in achieving emissions targets. Thus, renewable energy technologies face opportunities and challenges as a result of this desire for 'accelerated innovation' $[2,3]$.

Since the need for accelerating innovation on a wide range of new energy sources is becoming recognized, wave and tidal energy attract growing attention as part of future diverse and low carbon energy systems. Wave and tidal energy have the potential to be particularly valuable contributors to a low-carbon energy mix, having different geographic distribution than wind and solar, greater predictability, and less intermittency. Even if only a fraction of this huge resource could be exploited, wave and tidal energy offer a very significant untapped renewable.

A very important aspect when extracting the wave energy in a coastal location is to consider the wave energy converters (WEC) that is more appropriate for the particular conditions usually encountered in that area. On the other hand, due to some particularities (as for example the bathymetry) the expected wave energy can vary considerably in a relatively small geographical scale, and the locations where the wave energy is usually higher than in the neighboring marine sectors are called hot spots. From this perspective, the main objective of the present work is to assess the performances of various existent WEC types that would operate in the nearshore. Three different groups of coastal environments were considered: the western Iberian nearshore, islands and the sea environments, respectively.

Various classifications of the existent wave energy converters have been made, but probably the most relevant are: after the location (shoreline, nearshore and offshore), after the operating principle (oscillating water column, overtopping devices and wave activated bodies) and after the directional characteristics (point absorber, terminator and attenuator). Since the present work targets especially the nearshore and offshore areas, the following converters were considered: Oceantec [4], Pelamis [5], Pontoon Power Converter [6], Seabased AB [6], Wave Dragon [7], Aqua Buoy [8], Archimedes Wave Swing [9], Langlee [6], OE Buoy [6] and Wavebob [6]. The rating powers and some other characteristics of these devices are given in Table 1. For each converter the manufacturer provided the nominal power (or power rating, which is the maximum power to be used with that device) and the power matrix that indicates the electric power (in $\mathrm{kw}$ ) expected to be provided by the device when working in the wave conditions that correspond to each specific sea state. The sea states are usually structured into bins of $0.5 \mathrm{~s} \times 0.5 \mathrm{~m}\left(\Delta T_{\mathrm{e}} \times \Delta H_{\mathrm{s}}\right)$. 
Thus, in order to assess correctly the expected electric power produced by a certain device in a specific location, a very important issue is to determine properly the wave energy distribution along the sea states and design diagrams for the bivariate distribution of the sea occurrences, which correspond to the sea states defined by significant wave height and energy period.

Table 1. Comparison of some basic characteristics of the devices considered in this study.

\begin{tabular}{lllll}
\hline Device & $\begin{array}{l}\text { Power per } \\
\text { unit }(\mathrm{kW})\end{array}$ & Movement & $\begin{array}{c}\text { Depth } \\
(\mathrm{m})\end{array}$ & Size \\
\hline Oceantec & 500 & heave & $30-50$ & medium \\
Pelamis & 750 & surge \& heave & $50-70$ & medium \\
P P Converter & 3620 & heave & deep & large \\
Seabased AB & 15 & heave & $30-50$ & small \\
Wave Dragon & 7000 & overtopping & $30-50$ & large \\
Aqua Buoy & 250 & heave & $>50$ & small \\
AWS & 2320 & heave & $40-100$ & medium \\
Langlee & 1665 & oscilating flaps & deep & medium \\
OE Buoy & 2800 & oscilating column & deep & medium \\
Wavebob & 1000 & heave & deep & medium \\
\hline
\end{tabular}

Medium to long term wave data time series, coming from either wave modelling systems or in situ measurements are considered for each coastal location in order to generate the scatter diagrams of the $H_{\mathrm{s}}-T_{\mathrm{e}}$ joint distributions. Such a diagram presents the probabilities of occurrences of different sea states expressed in percentages from the total number of occurrences and the color of each bin indicates the percentage according to a color-map, which was designed the same for all diagrams and is illustrated in the color-bar of the figures. As an example, a bivariate diagram, designed in a location from the coastal environment in the north of Portugal, is presented in Figure 1 structured in total and winter time, respectively, where the winter time represents the periods between October to March.

The wave power isolines, which are represented in each diagram, have been computed using the equation of the deep water energy flux [10]:

$$
P_{W}=\frac{\rho g^{2}}{64 \pi} T_{e} H_{s}^{2},
$$

where $P_{W}$ is the energy flux in watts per meter of crest length, $\rho=1025 \mathrm{~kg} / \mathrm{m}^{3}$ is the density of sea water, $g$ is the acceleration of gravity.

As the diagram presented on the left side of Figure 1 shows, for the total time the bulk of the occurrences in terms of significant wave height is in the interval 1-3 meters, while as regards the energy periods, the interval 5.5-11 seconds appears to be dominant. The winter time conditions are illustrated in the right side of Figure 1, where it can be seen that the interval of the most probable wave energies moves with about two bins towards the higher values in each direction. Figure 1 also shows that for the total time, although most of the wave energy lies between the power isolines of 5 and $25 \mathrm{~kW} / \mathrm{m}$, significant occurrences can also be encountered in the interval between the insolence of 25 and $50 \mathrm{~kW} / \mathrm{m}$. In the winter time, the cluster of most probable occurrences moves from the interval 5$25 \mathrm{~kW} / \mathrm{m}$ to the interval $25-50 \mathrm{~kW} / \mathrm{m}$. In this case, some relevant occurrences can be seen also for the 
power interval $50-100 \mathrm{~kW} / \mathrm{m}$. The above diagrams show that the most probable sea states are concentrated along the diagonal that makes an angle of about 45 degrees with the horizontal. Such concentration of the sea states occurrences along a diagonal line appears to be higher in the winter time. To each bivariate diagram, a table was associated (not shown in the work), giving the wave activity during the time interval considered. The elements of these tables indicate the number of occurrences, in percentages from the total.

Figure 1. The specific pattern for the west Iberian nearshore, bivariate distributions of occurrences corresponding to the sea states defined by $H_{s}$ and $T_{e}$ as resulted from simulations with SWAN model, performed for the three-year time interval 2009-2011 (structured in total and winter time, respectively). The color scale is used to represent the contribution of the sea state to the total incident energy, as a percentage. The wave power isolines are also represented.
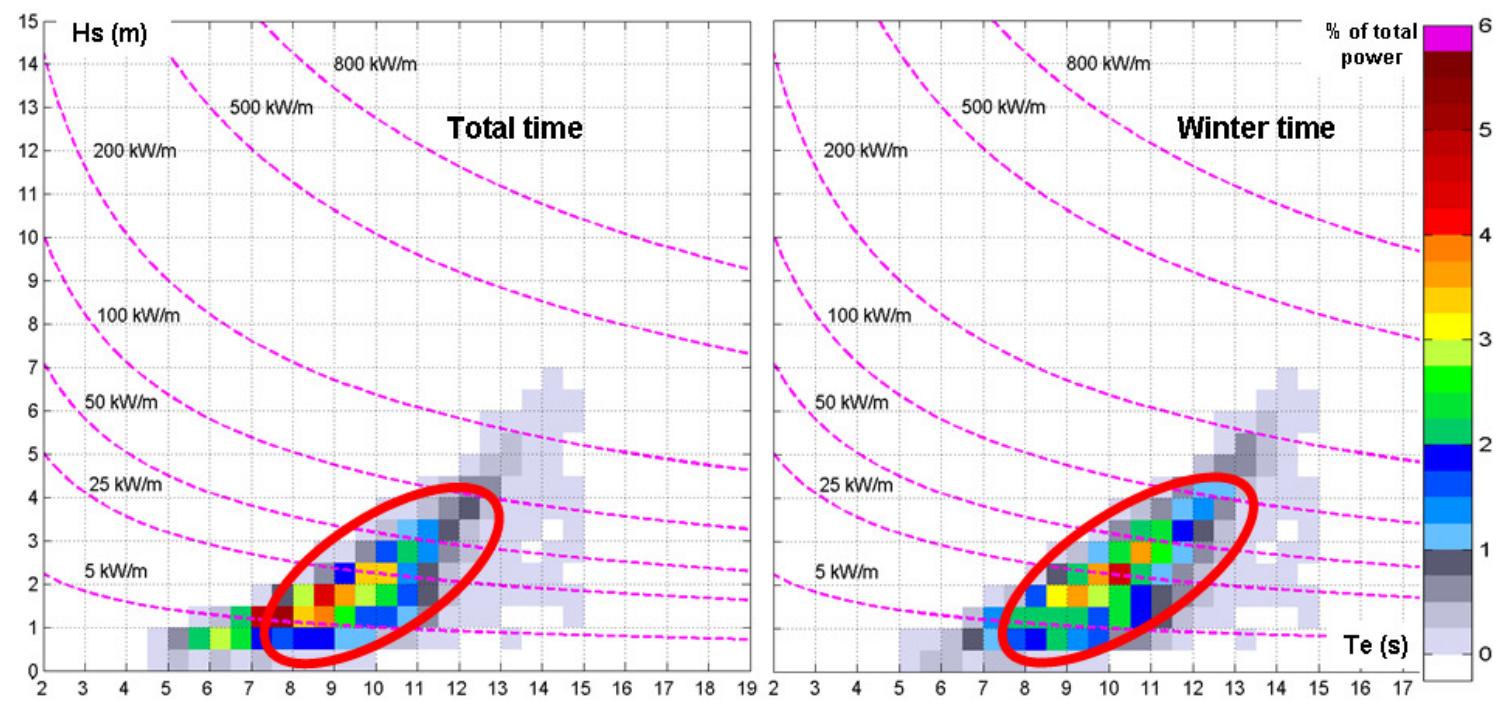

The approach considered to evaluate the electric power of a certain WEC in a specific site is to associate to the power matrix of each device the matrix that indicates the wave activity for the respective location in a determined time interval. This yields to the following formula for the estimation of the average electric power that might be expected in the time interval associated to the matrix that gives the wave activity:

$$
P_{E}=\frac{1}{100} \cdot \sum_{i=1}^{n_{T}} \sum_{j=1}^{n_{H}} p_{i j} \cdot P_{i j}
$$

where $p_{i j}$ represent the energy percents corresponding to the bin defined by the line $i$ and the column $j$ and $P_{i j}$ the electric power corresponding to the same energy bin for the WEC considered, $n_{T}$ indicates the number of bins on the horizontal (corresponding to the Te discretization) and $n_{H}$ is the number of bins on the vertical (corresponding to the $H s$ discretization). Thus, considering this approach, the average electric power expected at each location and for each device can be estimated with a certain approximation. The a detailed description of the approach considered in the evaluation of the expected wave power provided by a certain device is given in $[11,12]$. 
Finally, at the end of this section it has to be also highlighted that the large wave energy farms may also play an active role in the coastal protection. Some recent studies [13, 14] showed that nearshore currents, which are the main factor in driving the coastline dynamics, are sometimes even more sensitive than the waves to the nearshore energy extraction. This is explained by the fact that the wave farms induce relevant changes, not only to the wave heights, but also to the wave directions. Nevertheless, for each particular coastal environment all the aspects have to be carefully studied to assess also the potential effects of the marine power plants on the aquatic environment and life, because many times in the history, the humanity invented things that were thought to be positive but in a larger time scale brought more harm than benefits.

\section{Results and Discussion}

\subsection{Transformation of the wave energy in western Iberian nearshore}

The first target area is the western Iberian nearshore and the analysis is structured in two parts, the Spanish coastal environment (corresponding to the northwestern side of the Iberian nearshore) and the Portuguese nearshore.

In order to identify the most energetic locations in the Spanish nearshore, an analysis based on long term WAM [15] model simulations was performed considering a numerical data set composed of hindcast wind and wave data for a period of 41 years (1960-2000) with a three-hourly frequency provided by Puertos del Estado (the Spanish Ports Authority). Twelve reference points (denoted as the I-points) were considered for the wave data analysis and their locations are illustrated in Figure 1a.

Subsequently, for assessing better the spatial distribution patterns of the wave energy SWAN [16] simulations with a higher resolution in the geographical space were also performed [17] and the background of Figure 1a presents the distribution of the mean relative wave power for a representative situation corresponding to the time frame 2010/01/16/h18 that reflect high non storm energy conditions.

The non dimensional normalized wave power is expressed as:

$$
P_{W n}=\frac{P_{W}}{P_{W \max }},
$$

where $P_{W}$ represents the wave power in the respective point of the computational domain and $P_{W \max }$ represents the maximum value, which for the cases considered in Figure 1 (a, b and c) is 100kW.

The average values of the wave power in the I-points, structured in total time (TT) and winter time (WT) are presented in Table 2, while Table 3 shows the expected electric power for the six most energetic reference points (I1, I2, I3, I5, I6 and I7), evaluations carried out considering the approach defined in the previous section, corresponding to the performance characteristics of five different WEC devices (Oceantec, Pelamis, Pontoon Power Converter, Seabased AB and Wave Dragon). Detailed descriptions of the way how these results were obtained together with some additional comments are given in [18]. 
Figure 2. Distribution of the mean relative wave power and the positions of the reference points in the northwest Iberian and Portuguese coastal environments (a) the northwestern Iberian nearshore (denoted as the Spanish nearshore), SWAN simulation corresponding to the time frame 2010/01/16/h18 reflecting high non storm energy conditions, the positions of 12 reference points are also indicated (I-points), the computational domains considered in the Portuguese nearshore are also indicated. (b) and (c) the northern and central domains defined in the Portuguese nearshore, SWAN simulations corresponding to the time frame 2010/04/22h18 reflecting average wave energy conditions, the positions of 15 reference points are defined in each case (NP and CP-points, respectively).

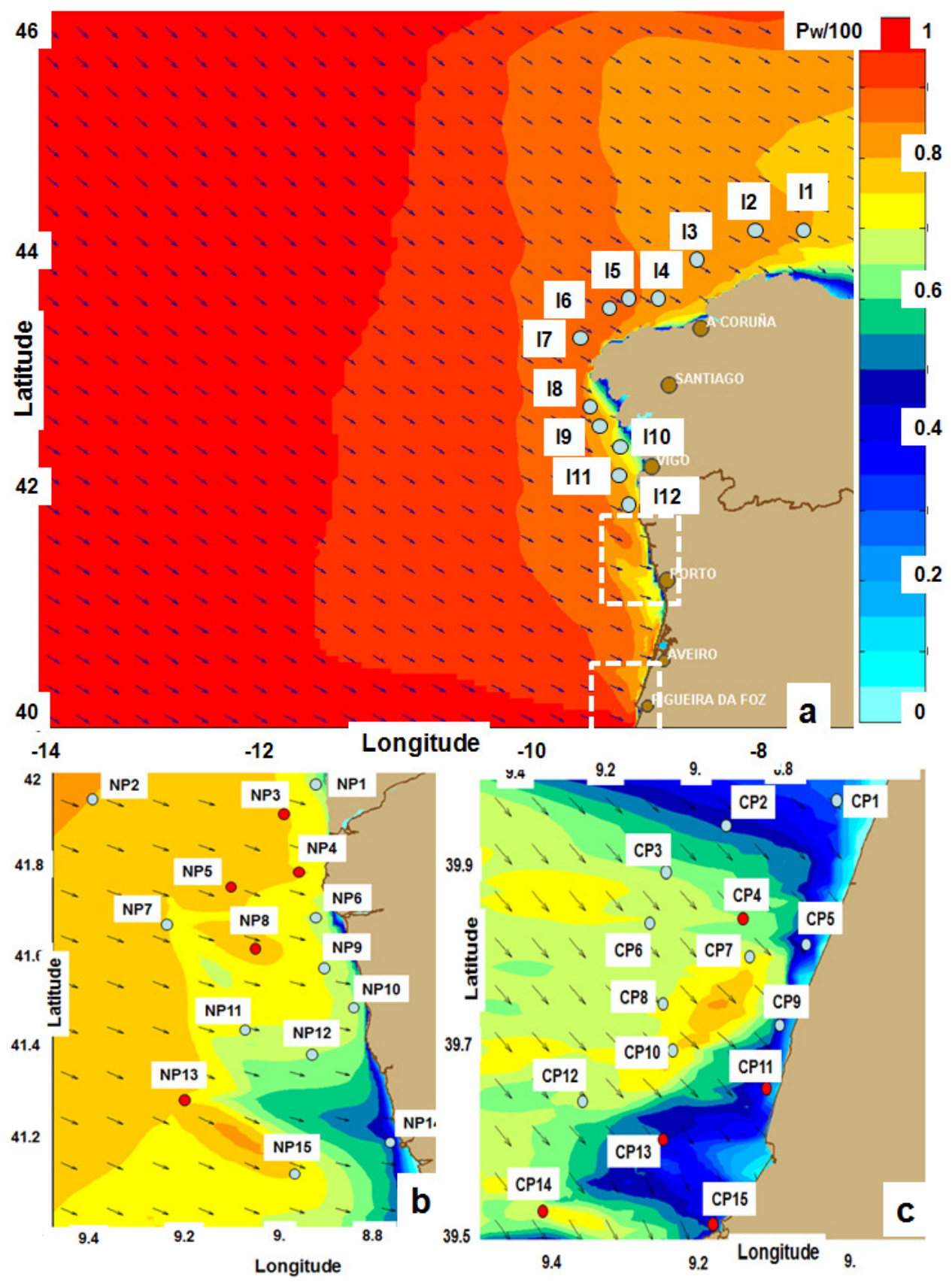


Table 2. Spanish nearshore (the I-points), average values of the wave power (structured in total TT and winter time WT, respectively), WAM model results for a 41-year period (1960-2000)

\begin{tabular}{|c|c|c|c|c|c|c|c|c|c|c|c|c|c|}
\hline Points & & I1 & I2 & $\mathrm{I} 3$ & I4 & I5 & I6 & I7 & I8 & I9 & I10 & I11 & I12 \\
\hline$P_{\text {wmed }}$ & TT & 28.7 & 29.7 & 29.2 & 25.5 & 28.7 & 31.7 & 30.2 & 20.4 & 23.8 & 12.8 & 15.7 & 19.4 \\
\hline $\mathrm{kW} / \mathrm{m}$ & WT & 51.9 & 53.6 & 52.3 & 44.6 & 50.9 & 56.2 & 53.3 & 36.8 & 42.3 & 25.0 & 30.1 & 34.2 \\
\hline
\end{tabular}

Table 3. Spanish nearshore, average electric power in $\mathrm{kW}$ for the six most energetic reference points studied, estimations corresponding to the characteristics of five different WEC devices.

\begin{tabular}{|c|c|c|c|c|c|c|c|c|c|c|}
\hline Point/ & Ocean & & Pelami & & Pontoo & ower C & Seal & & Wave D & son \\
\hline Period & $\mathrm{TT}$ & WT & TT & WT & TT & WT & TT & WT & TT & WT \\
\hline$\overline{\mathrm{I} 1}$ & 96.5 & 102.8 & 114.2 & 145.4 & 221.5 & 252.1 & 2.6 & 3.2 & 2037.2 & 2667.6 \\
\hline $\mathrm{I} 2$ & 99.9 & 105.4 & 118.1 & 150.2 & 227.2 & 258.5 & 2.7 & 3.3 & 2083.8 & 2730.9 \\
\hline I3 & 97.2 & 100.4 & 118.4 & 150.0 & 224.8 & 253.1 & 2.7 & 3.2 & 2112.6 & 2768.6 \\
\hline I5 & 94.4 & 94.4 & 115.6 & 144.8 & 219.7 & 244.4 & 2.6 & 3.2 & 2095.2 & 2736.4 \\
\hline I6 & 107.1 & 106.5 & 126.6 & 158.6 & 239.4 & 239.4 & 2.8 & 3.4 & 2197.8 & 2875.0 \\
\hline I7 & 105.0 & 103.6 & 124.5 & 155.9 & 236.3 & 263.7 & 2.8 & 3.3 & 2172.4 & 2839.5 \\
\hline
\end{tabular}

As regards the second part of the first target area, represented by the Portuguese continental coastal environment, it has to highlight first that since this was a very popular area for the wave energy developers various studies have been previously performed as for example those presented in [19] and [20]. Moreover, some wave forecasting systems, as that described in [21] are operational and they can provide a large database for extended analyses of the wave conditions.

The results analyzed at this level of the work are based on medium term simulations carried out with a different wave prediction system than in [21]. This uses Wave Watch III [22], for the wave generation at the scale of the entire North Atlantic Ocean, and SWAN, for the coastal wave transformation. The results provided by this system were evaluated against measurements coming from two directional buoys. Pursuing the wave energy patterns, especially as regards the most frequent wave power spatial distributions in the two medium resolution computational domains, fifteen reference points were defined for each computational domain and their positions are indicated in Figures $1 \mathrm{~b}$ and 1c, for the northern and central computational domains. They will be denoted as NP (northern points) for and CP (central points), respectively. The background of the figures illustrates the distribution of the normalized wave power in the two medium resolution computational domains, simulations for the time frame 2010/04/22h18. The above situation corresponds to average wave energy conditions that are in line with the most usual wave energy patterns encountered in the target areas considered.

The average values of the wave power in the NP and CP-points, also structured in total and winter time are presented in Table 4. 
Table 4. Portuguese nearshore, average values of the wave power resulted from simulations with SWAN performed for the three-year time interval 2009-2011, structured in total TT and winter time WT, respectively.

\begin{tabular}{|c|c|c|c|c|c|c|c|c|c|c|c|c|c|c|c|}
\hline NP-points & NP1 & NP2 & NP3 & NP4 & NP5 & NP6 & NP7 & NP8 & NP9 & NP10 & NP11 & NP12 & NP13 & NP14 & NP15 \\
\hline TT & 17.3 & 27.8 & 26.2 & 22.9 & 25.2 & 19.8 & 25.2 & 27.4 & 23.7 & 17.9 & 24.6 & 22.8 & 26.5 & 17.1 & 24.9 \\
\hline WT & 26.8 & 43.1 & 40.7 & 35.4 & 38.9 & 30.5 & 38.9 & 42.7 & 36.5 & 27.9 & 37.9 & 34.9 & 41.3 & 26.6 & 38.6 \\
\hline CP-points & $\mathrm{CP} 1$ & $\mathrm{CP} 2$ & $\mathrm{CP} 3$ & CP4 & CP5 & CP6 & CP7 & CP8 & CP9 & CP10 & CP11 & CP12 & CP13 & CP14 & CP15 \\
\hline TT & 20.3 & 24.7 & 23.2 & 24.9 & 21.7 & 24.1 & 23.4 & 23.5 & 21.2 & 24.9 & 24.8 & 25.3 & 24.5 & 24.8 & 24.8 \\
\hline WT & 31.5 & 38.4 & 35.9 & 38.9 & 33.7 & 37.5 & 36.3 & 36.4 & 32.8 & 36.1 & 38.9 & 39.1 & 37.8 & 38.5 & 38.5 \\
\hline
\end{tabular}

Table 5 shows the expected electric power for the five most energetic reference points in each computational domain (NP3, NP4, NP5, NP8, NP13 and CP4, CP11, CP13, CP14, CP15, respectively), corresponding to four different wave converters (Pelamis, Archimedes Wave Swing, Aqua Buoy and Wave Dragon). Some more information concerning these results is provided in [11].

Table 5. Portuguese nearshore, average electric power in $\mathrm{kW}$ for ten most energetic reference points, estimations corresponding to the characteristics of four different WEC devices.

\begin{tabular}{lllllllll}
\hline Point/ & Pelamis & \multicolumn{3}{c}{ AWS } & \multicolumn{3}{c}{ Aqua Buoy } & \multicolumn{2}{c}{ Wave Dragon } \\
\cline { 2 - 8 } Period & TT & WT & TT & WT & TT & WT & TT & WT \\
\hline NP3 & 95.1 & 130 & 282.7 & 446 & 34.4 & 48.9 & 907.5 & 1359 \\
NP4 & 78.7 & 109 & 246.9 & 396 & 28.8 & 41.5 & 766.7 & 1153 \\
NP5 & 98.0 & 134 & 286.8 & 451 & 35.7 & 50.6 & 927.9 & 1388 \\
NP8 & 101.1 & 139 & 302.8 & 476 & 36.3 & 51.4 & 979.2 & 1475 \\
NP13 & 100.2 & 138 & 295.9 & 467 & 36.1 & 51.4 & 957.1 & 1440 \\
CP4 & 95.8 & 132 & 274.5 & 436 & 34.1 & 48.6 & 895.8 & 1339 \\
CP11 & 85.4 & 121 & 275.1 & 447 & 30.3 & 44.2 & 859.2 & 1317 \\
CP13 & 102.3 & 142 & 286.9 & 455 & 36.2 & 51.8 & 955.9 & 1436 \\
CP14 & 97.5 & 136 & 274.0 & 433 & 33.9 & 48.3 & 905.2 & 1354 \\
CP15 & 83.1 & 115 & 262.7 & 421 & 30.0 & 43.0 & 820.8 & 1232 \\
& & & & & & & & \\
\hline
\end{tabular}

\subsection{Transformation of the wave energy in the nearshore of Canary Islands and Madeira Archipelago}

For the next study, WANA data from Puertos de Estado have been used for the reason that they bring more information about the waves in the ocean and in the coastal areas neighboring the Canary Islands. WANA data are temporally series of wind and wave parameters provided by numerical models. They come from the prediction system that the Spanish Port Authority has been developed in collaboration with the Spanish National Agency for Meteorology (AEMET). It has to be however highlighted that these data are not simple predictions, but they are analysis or diagnosis data. To generate the wave fields, the models WAM and Wave Watch III have been used, along with the wind fields coming from the HIRLAM model.

Twelve reference points (C-points) were considered for the wave data analysis in the coastal area of the Canary Islands and their positions are illustrated in Figure 3a. For this level of the analysis, a seventeen-year time interval (1996-2012) is considered. 
The average values of the wave power in the C-points, also structured in total and winter time are presented in Table 6.

Table 6. Average values of the wave power: C-points, Canary Islands, structured, in total time (TT) and winter time (WT), respectively, data processed for the seventeen-year time interval 1996-2012; Mpoints, Madeira Archipelago (WT), representing results of SWAN simulations performed for the time intervals (07/10/1997e01/03/1998) and (01/12/2000e05/03/2001).

\begin{tabular}{cllllllllll}
\hline C-points & C1 & C2 & C3 & C4 & C5 & C6 & C7 & C8 & C9 & C10 \\
\hline TT & 16.3 & 16.2 & 17.3 & 17.3 & 16.8 & 16.9 & 17.4 & 19.1 & 19.1 & 19.3 \\
\hline WT & 18.2 & 18.4 & 19.2 & 19.0 & 18.3 & 18.5 & 19.2 & 20.6 & 20.6 & 20.7 \\
\hline M-points & M1 & M2 & M3 & & & & & & & \\
\cline { 1 - 3 } WT & 51.5 & 65.4 & 57.4 & & & & & & &
\end{tabular}

Figure 3. (a) The positions of the reference points considered in the area of the Canary Islands (C-points). The other data points available are represented with white; (b) Distribution of the mean relative wave power in the vicinity of Madeira island (SWAN simulation corresponding to the time frame 2001/03/15/h21, average energetic conditions), the position of the reference point M1 is also represented; (c) Wave energy pattern in the vicinity of Porto Santo island (SWAN simulation corresponding to the time frame 2001/03/15/h21, average energetic conditions), the position of the reference points M2 and M3 are also represented.

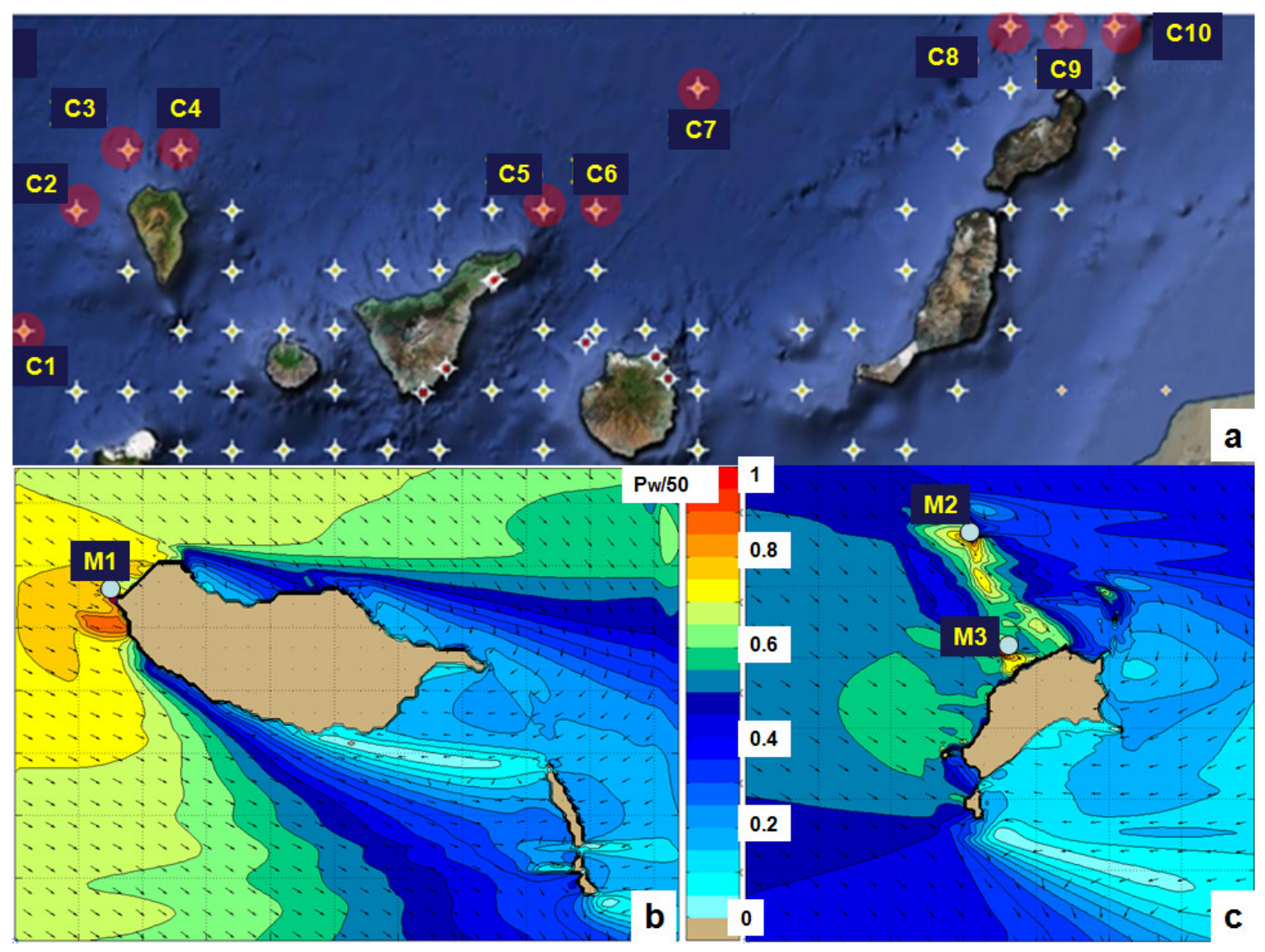


Table 7 shows the expected electric power corresponding to all these reference points for six wave energy converters that are considered more appropriate for such environment because they are designed to operate in deep water (Pelamis, Archimedes Wave Swing, Aqua Buoy, OE Buoy, Langlee and Wave Bob). A more detailed description of these results is given in [23].

Table 7. Canary Islands, average electric power in $\mathrm{kW}$ for the reference points studied, estimations corresponding to the characteristics of six different WEC devices.

\begin{tabular}{|c|c|c|c|c|c|c|c|c|c|c|c|c|}
\hline \multirow{2}{*}{$\begin{array}{l}\text { Point/ } \\
\text { Period }\end{array}$} & \multicolumn{2}{|c|}{ Pelamis } & \multicolumn{2}{|l|}{ AWS } & \multicolumn{2}{|c|}{ Aqua Buoy } & \multicolumn{2}{|c|}{ OE Buoy } & \multicolumn{2}{|c|}{ Langlee } & \multicolumn{2}{|c|}{ Wave Bob } \\
\hline & $\overline{\mathrm{TT}}$ & WT & TT & WT & TT & TT & WT & WT & TT & WT & TT & WT \\
\hline$\overline{\mathrm{C} 1}$ & 65.4 & 78.8 & 260.1 & 403.2 & 24.3 & 52.2 & 52.9 & 32.3 & 126.9 & 168.8 & 86.9 & 112.8 \\
\hline $\mathrm{C} 2$ & 66.2 & 79.5 & 260.2 & 403.0 & 24.6 & 52.1 & 53.0 & 32.5 & 128.2 & 171.2 & 87.4 & 113.4 \\
\hline $\mathrm{C} 3$ & 76.0 & 90.0 & 273.5 & 421.0 & 27.6 & 61.5 & 62.0 & 36.0 & 143.1 & 188.5 & 95.5 & 123.0 \\
\hline $\mathrm{C} 4$ & 74.8 & 88.3 & 271.8 & 418.5 & 27.2 & 60.5 & 60.6 & 35.4 & 141.1 & 185.5 & 94.4 & 121.3 \\
\hline $\mathrm{C} 5$ & 72.5 & 80.8 & 255.3 & 391.1 & 25.8 & 61.5 & 57.2 & 32.3 & 134.4 & 169.2 & 89.7 & 111.5 \\
\hline C6 & 74.1 & 82.1 & 257.7 & 394.0 & 32.3 & 63.1 & 58.2 & 32.7 & 137.1 & 171.8 & 91.3 & 112.9 \\
\hline $\mathrm{C} 7$ & 81.3 & 90.4 & 270.0 & 412.6 & 28.7 & 69.2 & 64.2 & 35.8 & 149.5 & 187.9 & 98.3 & 121.9 \\
\hline $\mathrm{C} 8$ & 87.8 & 93.4 & 275.6 & 417.1 & 30.7 & 75.6 & 66.3 & 37.0 & 159.6 & 193.7 & 103.7 & 124.9 \\
\hline C9 & 88.5 & 93.2 & 275.8 & 416.6 & 31.0 & 76.3 & 66.1 & 37.0 & 160.7 & 193.4 & 104.2 & 124.7 \\
\hline $\mathrm{C} 10$ & 90.3 & 94.2 & 277.4 & 418.1 & 31.5 & 78.3 & 67.1 & 37.3 & 163.6 & 195.5 & 105.7 & 125.7 \\
\hline
\end{tabular}

The next island environment considered is represented by Madeira Archipelago. Unlike the area of Canary Islands, this is composed of two major islands quite distanced (Madeira and Porto Santo) while southeast of Madeira, lies a group of smaller islands called the Desert Islands. The background of the Figures $3 b$ and $3 c$ illustrate the distribution of the normalized wave power in two medium resolution computational domains, corresponding to Madeira and Porto Santo, respectively, simulations corresponding to the time frame 2001/03/15/h21. The above situation corresponds to average wave energy conditions that are in line with the most usual wave energy patterns encountered in the target areas considered and the wave power was divided this time by $50 \mathrm{~kW}$. Three reference points (Mpoints) were selected, M1 in Madeira and M2 and M3 in Porto Santo, and they correspond to locations that have been identified as relevant hot spots with average energy considerably higher than the neighboring areas. The existence of such relevant hot spots in island environment has been also identified in the Archipelago of Azores, as presented in [24].

The average values of the wave power in the M-points, also structured in total and winter time are presented in Table 6. Table 8 gives the expected electric power for the reference points M1 and M3 corresponding to the wave converters (Pelamis, Aqua Buoy and Wave Dragon). More information concerning these results and the wave conditions and wave energy potential in Madeira Archipelago are provided in [12].

\subsection{Transformation of the wave energy in sea environment}

The next work direction is related to the study of the wave energy potential in sea environment and a representative case is given by two enclosed seas, the Black and the Caspian. The wave climate and energy in the Black sea was studied by various authors considering a modeling system SWAN based [25-27]. Following the above results, Figure 4a illustrates the distribution of the mean relative wave 
power in the Black Sea (divided by $20 \mathrm{~kW}$ ) as a result of a SWAN simulation corresponding to the time frame 1997/01/12/h12 and reflecting average energetic conditions.

The position of the reference point (B-point) is also represented. Figure $4 \mathrm{~b}$ illustrates a relevant pattern concerning the distribution of the mean relative wave power in the Caspian Sea, as a result of SWAN simulation corresponding to the time frame 2009/10/02/h18, winter average energy conditions [28]. As Figure 4b shows, the Caspian sea is in general poor in terms of wave energy with the exception of the centre of the sea (located in deep water) where some energy concentration occurs. As regards the Black Sea, the western side is more energetic. Moreover, this area appears to be also rich in wind energy resources [29], so it might become interesting from the perspective of the implementation of hybrid wind-wave projects. From this perspective, Table 8 presents the expected electric power (from the wave converters Pelamis, Aqua Buoy and Wave Dragon) for two reference points B, in the

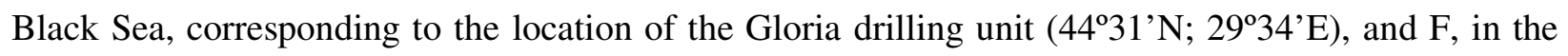
North Sea at the location Fino1 (54 ${ }^{\circ} 1^{\prime} N$; $\left.6^{\circ} 35^{\prime} \mathrm{E}\right)$ where a wind farm already operates [30]. In both places wave measurements are available for the time interval 2003-2009.

Figure 4. (a) Distribution of the mean relative wave power in the Black Sea (SWAN simulation corresponding to the time frame 1997/01/12/h12, average energetic conditions) and the position of the reference point (B-point); (b) Distribution of the mean relative wave power in the Caspian Sea (SWAN simulation corresponding to the time frame 2009/10/02/h18, winter average energy conditions).

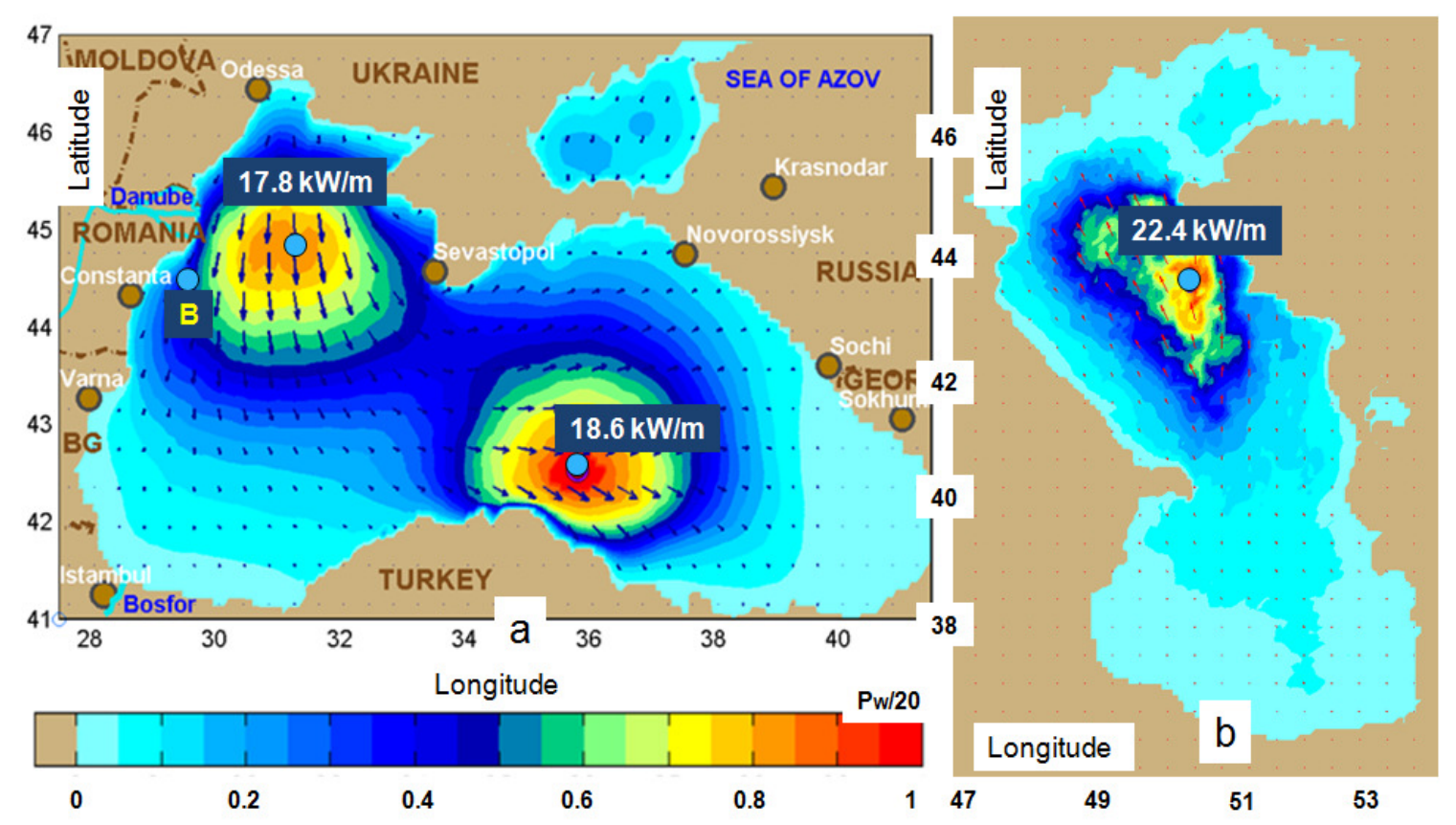


Table 8. Average electric power in $\mathrm{kW}$ for the converters: Pelamis, Aqua Buoy and Wave Dragon in two reference points in Madeira (M1 and M3), one reference point in the Black Sea (B-point) and another reference point in the North Sea at Fino station (F-point).

\begin{tabular}{lllllll}
\hline Point/ & Pelamis & \multicolumn{3}{c}{ Aqua Buoy } & \multicolumn{2}{c}{ Wave Dragon } \\
\cline { 2 - 7 } Period & TT & WT & TT & WT & TT & WT \\
\hline M1 & - & 134.9 & - & 40.2 & - & 1147.4 \\
M3 & - & 105.3 & - & 50.4 & - & 1644.2 \\
B & 60.0 & 89.0 & 15.9 & 24.0 & 391.2 & 578.1 \\
F & 69.7 & 98.0 & 12.1 & 25.5 & 735.4 & 895.1 \\
& & & & & & \\
\hline
\end{tabular}

\subsection{Discussions}

Eleven different wave energy converters were evaluated in the present work considering the specific wave conditions from various coastal environments. At this point, the discussion will be focused on three devices that were found from various points of view more appropriate in many coastal environments. These are: Aqua Buoy, Pelamis and Wave Dragon. Figure 5 ( $\mathrm{a}, \mathrm{b}$ and c) illustrates the range of the electric power expected from these devices in the coastal environments studied. As the results presented in the figure show, the electric power expected from a certain device varies in a relatively wide range depending on the location and also on the season (TT or WT). Thus, for Aqua Buoy this range is from $12 \mathrm{~kW}$ (TT) in the Black Sea to $52 \mathrm{~kW}$ (WT) in the Portuguese nearshore (with the observation that this device was not evaluated in the Spanish nearshore). For Pelamis, the range of the expected electric power is from about $60 \mathrm{~kW}$ (TT) in the Black Sea to $159 \mathrm{~kW}$ (WT) in the Spanish coastal environment while as regards the Wave Dragon the variation is even higher in both relative and absolute terms starting from $391 \mathrm{~kW}$ (TT) in the Black Sea and reaching $2875 \mathrm{~kW}$ (WT) in the Spanish nearshore.

Besides the expected values of the electric power, two different indicators of the efficiency of wave energy transformation into electricity were also evaluated. The first is the load factor defined as the average power capture divided by the device rating:

$$
I_{L}=100 \cdot P_{E} / P_{\text {nom }},
$$

For the same three devices (Pelamis, Aqua Buoy and Wave Dragon) the values of this index corresponding to the two extremes (the Spanish nearshore, which is the most energetic and the sea environment, which is the less energetic) are presented in Table 9. The results show that from this point of view the Wave Dragon appears to be the most effective device with a maximum value of the load factor of 48.7, in comparison with Pelamis for which the maximum value of this factor is only 21.1

The second indicator was denoted as the Wave Energy Transformation index $\left(I_{W E T}\right)$ [22] and gives the ratio of the average electric power to the average wave energy expected in a specific location:

$$
I_{W E T}=P_{E} / P \text {. }
$$

Such index can provide a comprehensive picture of the efficiency of different WECs in various sites and its values for the same three WECs are presented in Table 10. 
Figure 5. Expected electric power in various coastal environments. (a) Aqua Buoy; (b) Pelamis; (c) Wave Dragon.

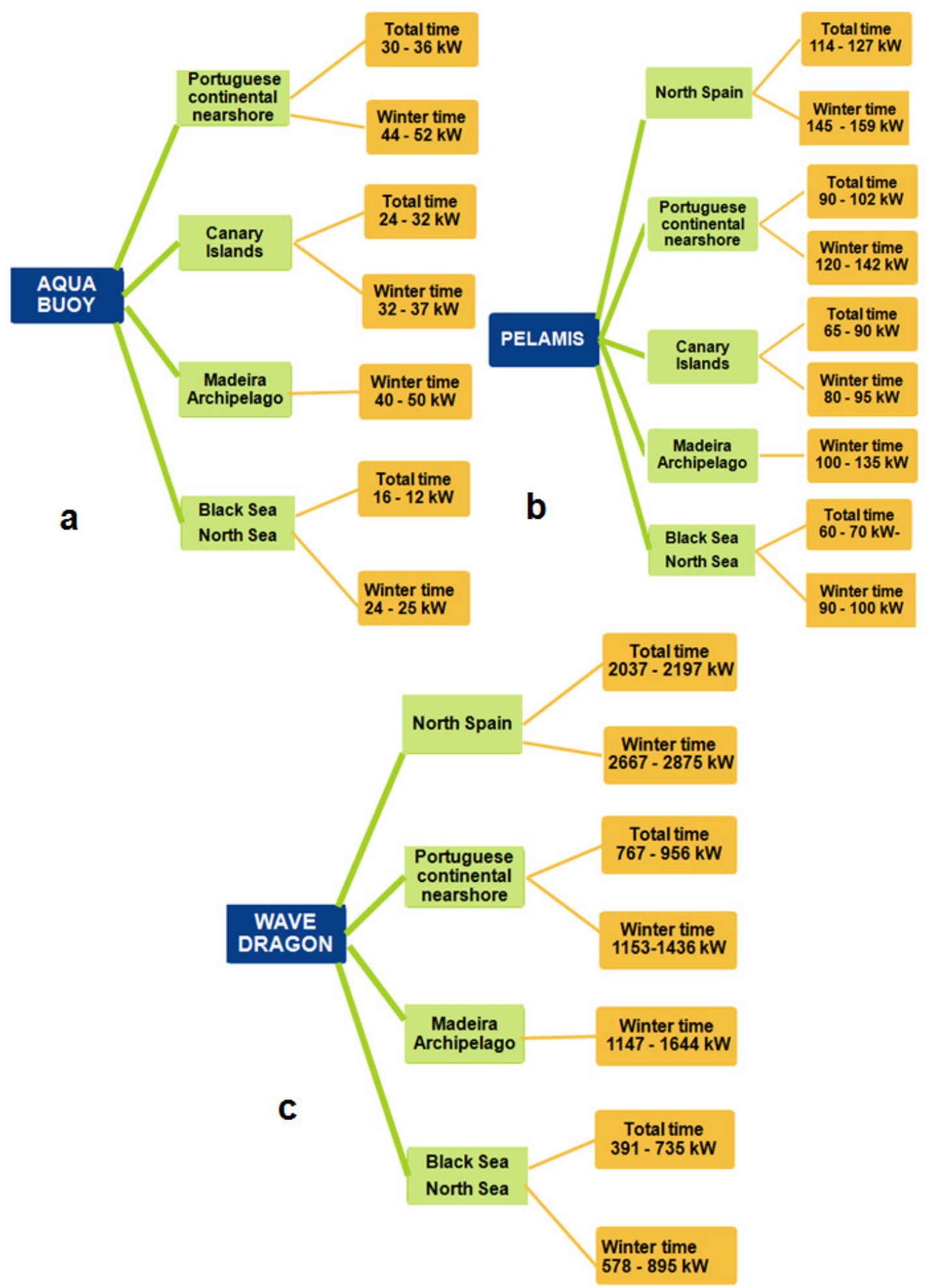


The range of variation of this index is also quite large from one environment to another. Thus, for Wave Dragon the WET index has values between 22.28 and 73.05, for Pelamis between 1.61 and 4.2, while for Aqua Buoy the range is [0.78-1.5].

Another observation coming from the analysis of the results presented in Table 10 would be that the values of this index are lower for WT in comparison with TT, which means that, although in winter time the wave energy is higher, the efficiency of its transformation into electricity is lower. This behavior might be explained through the fact that in winter the wave energy is more scattered along the sea states.

Table 9. Values of the load factor in the Spanish nearshore and in sea environment (the Black and the North seas). The WECs considered are: Pelamis, Aqua Buoy and Wave Dragon.

\begin{tabular}{lllllll}
\hline $\begin{array}{l}\text { Point/ } \\
\text { Period }\end{array}$ & Pelamis & \multicolumn{3}{c}{ Aqua Buoy } & \multicolumn{2}{c}{ Wave Dragon } \\
\hline & TT & WT & TT & WT & TT & WT \\
$\begin{array}{l}\text { Spanish } \\
\text { nearshore }\end{array}$ & $15.2-16.9$ & $19.3-21.1$ & - & - & $34.5-37.3$ & $45.2-48.7$ \\
$\begin{array}{l}\text { Sea } \\
\text { environment }\end{array}$ & - & $11.9-13.07$ & - & $8.4-9.5$ & - & $8.2-2.78$ \\
\hline
\end{tabular}

Table 10. Values of the index $I_{\text {wet }}$ in the Iberian nearshore (Spanish and Portuguese) and in Madeira archipelago. The WECs considered are Pelamis, Aqua Buoy and Wave Dragon.

\begin{tabular}{lllllll}
\hline $\begin{array}{l}\text { Point/ } \\
\text { Period }\end{array}$ & Pelamis & \multicolumn{3}{l}{ Aqua Buoy } & \multicolumn{3}{l}{ Wave Dragon } \\
\hline & TT & WT & TT & WT & TT & WT \\
$\begin{array}{l}\text { Spanish } \\
\text { nearshore }\end{array}$ & $3.98-4.13$ & $2.80-2.93$ & - & - & $69.42-73.05$ & $51.0-53.77$ \\
$\begin{array}{l}\text { Portuguese } \\
\text { Nearshore }\end{array}$ & $3.3-4.2$ & $3.0-3.7$ & $1.2-1.5$ & $1.1-1.4$ & $33.1-39.2$ & $32.0-38.0$ \\
$\begin{array}{l}\text { Madeira } \\
\text { archipelago }\end{array}$ & - & $1.61-2.62$ & - & $0.77-0.78$ & - & $22.28-25.14$ \\
\hline
\end{tabular}

\section{Conclusions}

The present work provides information about the wave conditions and on the efficiency of the wave energy transformations in three different types of coastal environment: continental coasts of the ocean (the western Iberian nearshore), island environment (Canary Island and Madeira Archipelago) and sea environment (Black, Caspian and North seas). More types of WECs were evaluated covering a large range from the existent nearshore and offshore devices.

The results show that only the evaluation of the wave energy in a certain location is not enough and even more important is in fact the correlation between the power matrix of a wave energy converter and the scatter diagram that gives the bivariate distribution of the sea states specific to the coastal area where the WEC is going to operate. From this perspective, a significant conclusion resulting from the 
present work is that a correct assessment of the expected distribution of the sea states in a certain location, reflected by the scatter diagrams that give the bivariate distributions ( $H s-T e)$, becomes an issue of extreme importance. From this perspective, although the number one design consideration for the WEC manufacturers is still represented by the survival issues, a future research direction to be taken into consideration would be to design converters with adjustable power matrices in order to fit better the environmental conditions from the locations where they will be installed and to increase in this way the efficiency of the wave energy transformation into electricity. On the other hand, if such a variable dynamics would blow the cost away and increase the risk of failure the alternative solution remains to select the most effective device from the point of view of the correspondence between its power matrix and the expected distribution of the sea states from a certain location.

Finally, as regards the sea environment, although it becomes quite obvious that most of the existing WEC devices are designed especially for the oceanic coastal environment, where the waves are more powerful and the wave periods are larger, the issue of the wave energy cannot be completely ignored. Hybrid wind-wave projects appear to be more meaningful for such conditions.

\section{Acknowledgments}

This work was supported by a grant from the Romanian Ministry of National Education, CNCS UEFISCDI, project number PN-II-ID-PCE-2012-4-0089 (project DAMWAVE).

\section{Conflicts of Interest}

The author declares no conflict of interest.

\section{References}

1. Nielsen K. Ocean Energy Technology Study. DanWEC; Technical Report No.1 for The Alliance for Offshore Renewables, 2012.

2. European Commission. Energy Roadmap 2050. 2011, COM (2011) 885.

3. European Commission. Technology Map of the SET-Plan. 2011; EC JRC-SETIS, EUR 24979.

4. EKINBERRI. OCEANTEC - Desarrollo tecnologico de un sistema de aprovechamiento de la energia de las olas. 2006; OCEANTEC - Informe publicable.

5. Henderson, R. Design, simulation, and testing of a novel hydraulic power take-off system for the Pelamis wave energy converter. Energy, 2006, 31, 271-283.

6. Babarit, A.; Hals, J.; Muliawan, M.J.; Kurniawan, A.; Moan, T.; Krokstad. J. Numerical benchmarking study of a selection of wave energy converters. Renewable Energy,, 2012, 41, 4463.

7. Kofoed, J.P.; Frigaard, P.; Friis-Madsen, E.; Sørensen, H.C. Prototype testing of the wave energy converter Wave Dragon. Energy, 2006, 31, 181-189.

8. Weinstein, A.; Fredrikson, G.; Parks, M.J.; Neislen, K. Aqua Buoy-The offshore wave energy converter: Numerical modeling and optimization. Proceedings of Oceans '04 MTS/IEEE TechnoOcean '04, Kobe, Japan, November 2004.

9. Environmental Change Institute, Variability of UK marine resources, 2005. 
10. Waters, R.; Engstrom, J.; Isberg, J.; Leijon, M. Wave climate off the Swedish west coast.. Renewable Energy 2009, 34, 1600-1606.

11. Silva, D.; Rusu, E.; Guedes Soares, C. Evaluation of Various Technologies for Wave Energy Conversion in the Portuguese Nearshore. Energies, 2013, 6(3), 1344-1364.

12. Rusu, E.; Guedes Soares, C. Wave energy pattern around the Madeira islands. Energy 2012, 5 , 771-785.

13. Diaconu, S.; Rusu, E.The environmental impact of a Wave Dragon array operating in the Black Sea. The Scientific World Journal, 2013,Article ID 498013, 1-20.

14. Rusu, E.; Guedes Soares, C. Coastal impact induced by a Pelamis wave farm operating in the Portuguese nearshore. Renewable Energy 2013, 58, 34-49.

15. WAMDI Group. The WAM model - A third generation ocean wave prediction model. J. Phys. Oceanogr. 1988, 18, 1775-1810.

16. Booij, N.; Ris, R.C.; Holthuijsen, L.H. A third-generation wave model for coastal regions. IModel description and validation. J. Geophys. Res. 1999, 104, 7649-7666.

17. Rusu, E.; Ventura Soares, C.; Rusu, L. Computational Strategies and Visualization Techniques for the Waves Modeling in the Portuguese Nearshore, Maritime Transportation and Exploitation of Ocean and Coastal Resources, 2006, Taylor \& Francis publications, London, , Vol II, pp. 11291136.

18. Morales Vaquero, A.; Castro Ruiz, F.; Rusu, E.Evaluation of the wave power potential in the northwestern side of the Iberian nearshore, Developments in Maritime Transportation and Exploitation of Sea Resources - Guedes Soares \& López Peña, 2014 Taylor \& Francis Group, London, , pp 1012-1019.

19. Rusu, E.;Guedes Soares, C. Numerical modeling to estimate the spatial distribution of the wave energy in the Portuguese nearshore. Renewable Energy, 2009, 34 (6), 1501-1516.

20. Rusu, E.; Gonçalves, M.; Guedes Soares, C. Evaluation of the wave transformation in an open bay. Ocean Engineering 2011, 38 (16), 1763-1781.

21. Guedes Soares, C.; Rusu, L.; Bernardino, M.; Pilar, P. An operational wave forecasting system for the Portuguese continental coastal area. J. Oper. Oceanogr. 2011, 4, 17-27.

22. Tolman, H.L. A third-generation model for wind waves on slowly varying, unsteady and inhomogeneous depths and currents. J. Phys. Oceanogr. 1991, 21, 782-797.

23. Molina Andres, O.; Castro Ruiz, F.; Rusu, L. Efficiency assessments for different WEC types in the Canary Islands Developments in Maritime Transportation and Exploitation of Sea Resources Guedes Soares \& López Peña, 2014 Taylor \& Francis Group, London, , Vol 2, pp 879-887.

24. Rusu, L.; Guedes Soares, C. Wave energy assessments in the Azores islands. Renew. Energy 2012, 45, 183-196.

25. Rusu, E. Strategies in using numerical wave models in ocean/coastal applications. Journal of Marine Science and Technology- Taiwan, 2011, 19,(1), 58-73.

26. Rusu, E. Wave energy assessments in the Black Sea. Journal of Marine Science and Technology, Springer, 2009, 14, (3), 359-372.

27. Rusu, L. Application of numerical models to evaluate oil spills propagation in the coastal environment of the Black Sea. Journal of Environmental Engineering and Landscape Management, 2010, 18 (4), 288-295. 
28. Rusu, E.; Onea, F. Evaluation of the wind and wave energy along the Caspian Sea. Energy, 2013, 50, 1-14.

29. Onea, F.; Rusu E. Evaluation of the Wind Energy in the North-West of the Black Sea. International Journal of Green Energy, 2014, 11(5), 465-487.

30. Onea, F. Study concerning the opportunity of the renewable energy extraction in marine environment with application to the Black Sea basin, PhD Thesis, Dunarea de Jos University of Galati, 2013, 223p.

(C) 2014 by the authors; licensee MDPI, Basel, Switzerland. This article is an open access article distributed under the terms and conditions of the Creative Commons Attribution license (http://creativecommons.org/licenses/by/3.0/). 